\title{
GAUGE APPROACH TO THE SPECIFIC HEAT IN THE NORMAL STATE OF CUPRATES
}

\author{
P.A. Marchetti \\ Dipartimento di Fisica "G. Galilei", University of Padova INFN, I-35131 Padova, Italy
}

A. Ambrosetti

Dipartimento di Fisica, INFN, University of Trento I-38100 Povo (TN), Italy

(Dated: November 4, 2018)

\begin{abstract}
Many experimental features of the electronic specific heat and entropy of high Tc cuprates in the normal state, including the nontrivial temperature dependence of the specific heat coefficient $\gamma$ and negative intercept of the extrapolated entropy to $T=0$ for underdoped cuprates, are reproduced using the spin-charge gauge approach to the $t-J$ model. The entropy turns out to be basically due to fermionic excitations, but with a temperature dependence of the specific heat coefficient controlled by fluctuations of a gauge field coupling them to gapful bosonic excitations. In particular the negative intercept of the extrapolated entropy at $T=0$ in the pseudogap "phase" is attributed to the scalar component of the gauge field, which implements the local no-double occupancy constraint.

PACS numbers: $71.10 . H f, 11.15 .-\mathrm{q}, 71.27 .+\mathrm{a}$
\end{abstract}

The low-temperature electronic entropy of high $T_{c}$ hole-doped cuprates in the normal ( "metallic") state exhibits a behavior rather unusual for a metal: the specific heat coefficient $\gamma$ which should be constant shows a non trivial temperature dependence [1] and even more spectacularly the $T=0$ intercept of the entropy, extrapolated from the approximate linear behavior at moderate temperatures, turns out to be negative in the underdoped region [2]. In this paper we apply the spin-charge gauge approach developed in $[3,4,5,5]$ to extract from the two-dimensional $t-J$ model the low temperature entropy and specific heat in the normal state and compare our results with the experimental data, in particular showing how this approach can explain the peculiar behavior mentioned above.

Let us first outline the main features of the experiments [1, 2, 7], following Ref. 1. In the normal state of $\mathrm{La}_{2-\delta} \mathrm{Sr}_{\delta} \mathrm{CuO}_{4}$ (LSCO) and $\mathrm{YBa}_{2} \mathrm{Cu}_{3} \mathrm{O}_{6+x}(\mathrm{YBCO})$ the electronic specific heat coefficient $\gamma=C^{e l} / T$ as a function doping concentration $\delta$ and temperature up to 250$300 \mathrm{~K}$ exhibits the following behavior. For strongly underdoped samples $\gamma(\delta, T) \sim \Delta \gamma^{L T}+B(\delta, T)$ where $\Delta \gamma^{L T}$ is a Low-Temperature upturn, $B(\delta, T)$ is slowly increasing roughly linearly in $\delta$ and $T$ with almost $\delta$-independent slope. At higher dopings the Low-Temperature upturn disappears and the samples become superconducting. The increasing part remains, but it saturates to a broad maximum at $T_{\gamma}^{*}$, followed by a slow decrease in $T . T_{\gamma}^{*}$ roughly coincides with the pseudogap temperature $T^{*}$ identified by the inflection point in the in-plane resistivity, as can be checked using the data on curvature of resistivity [8], see Fig 1 . In the decreasing region $\gamma$ becomes almost $\delta$-independent. Similar features are exhibited, in the appropriate doping range, also by $\mathrm{Bi}_{2} \mathrm{Sr}_{2} \mathrm{CaCu}_{2} \mathrm{O}_{8+x}$ (Bi2212) data 9], so they can thus be considered as rather generic in cuprates and therefore it is reasonable to

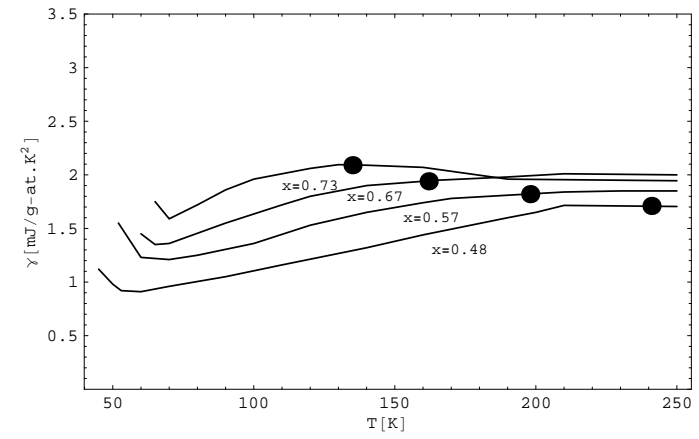

FIG. 1: The dots mark the inflection point on resistivity $\left(\mathrm{T}^{*}\right)$ as given in [8] on $\gamma$ data in $\mathrm{YBa}_{2} \mathrm{Cu}_{3} \mathrm{O}_{6+x}$ taken from [9]; semiempirically $\delta \approx 0.2 x$, see [9].

explore a physical interpretation in terms of the physics of doped Mott insulator described by the $t-J$ model.

An attempt to discuss the specific heat coefficient in terms of $S U(2)$ slave boson theory [10] of the $t-J$ model appears in Ref 11, obtaining for the gauge contribution $\gamma \approx-T \ln T$ and some data are fitted with this formula. Further attempts to understand the behavior of the specific heat can be found in 12] 13].

In spite of the complex features of $\gamma(\delta, T)$ described above, the entropy $S(\delta, T)$ of the normal state exhibits a simpler continuity: for low dopings it is approximately linear in $T$ with increasing slope as $\delta$ increases, at higher $T$ the slope becomes almost $\delta$-independent with increasing but negative intercept at $T=0$. At higher dopings $(\delta \gtrsim 0.19)$ also the intercept becomes almost $\delta$ independent and approximately 0 . On the basis of the above continuity it was argued in Ref. 1 that in YBCO the spin excitations may be relevant for all $\delta$ and the above results are better described as a modification of the low-energy spin spectrum as $\delta$ changes than by a 
simple band model. The spin-charge gauge approach appears to partially substantiate such claims. The negative intercept of the entropy suggests a negative contribution to entropy of a "constraint-field" , which acts reducing the low-energy degrees of freedom or more precisely removing them from the temperature/energy region considered. This was proposed in the analysis of the thermodynamics of the $t-J$ model performed in [14] within the slave-boson approach. In fact, a negative contribution to entropy naturally arises in a gauge approach from the scalar component of the gauge field (in the Coulomb gauge) enforcing "Gauss law". Let us now sketch the basis of the spin-charge gauge approach and its application to the computation of entropy and specific heat.

This approach assumes as a (simplified) model for $\mathrm{CuO}$ layers in high $T_{c}$ cuprates the $2 \mathrm{D} t-J$ model with $t / J \sim 3$. Neglecting $t^{\prime}, t^{\prime \prime}$, details of Fermi surface (FS) are lost but the analysis is simplified, hopefully retaining the basic relevant features. The model is treated in an "improved Mean Field Approximation"(MFA) via a gauge theory of spin-charge decomposition, obtained by gauging the global spin and charge symmetries of the model [15]. This gauging is obtained introducing spin and charge ChernSimons gauge fields. The nice feature of introducing these gauge fields is the possibility of a more flexible treatment of charge and spin responses within a a spin-charge decomposition scheme. In the end they will disappear from the game in MFA, but leaving behind sign of their presence crucial for the low-energy physics, as discussed below.

The basic fields adopted in this approach for the spincharge decomposition of the $t-J$ model are a charged spinless fermion, the holon [16], a neutral spin $1 / 2$ boson of a non-linear $\sigma\left(C P^{1}\right)$ model, the spinon, and a slave-particle gauge field (not to be confused with spin and charge Chern-Simons gauge fields). The spin-gauge field in MFA attaches spin vortices to the empty-site positions. The spinons moving across this gas of vortices acquire a mass gap, with a theoretically derived doping dependence, $m_{s} \sim \sqrt{|\delta \ln \delta|}$ consistent with AF correlation length at small $\delta$ derived from neutron experiments [17]. In MFA at low temperature and small doping concentration, in the parameter region to be compared with the "pseudogap phase" (PG) of the cuprates, the holons move in a statistical magnetic field with flux $\pi$ per plaquette generated by the charge-gauge field. This "phase" shares some similarity with the $\pi$-flux phase appearing in the slave boson formalism [18].

Around the pseudogap temperature $T^{*}$ the $\pi$-flux lattice "melts" and we enter in the "strange metal phase" (SM), at higher $\delta$ or $T$, see Fig. 2. Notice that, since only holons are involved and not full electrons, this is not a true phase transition as the one appearing in the DDW formalism [19].

In PG, as a consequence of the $\pi$-flux, the holons are converted via Hofstadter mechanism into two species of

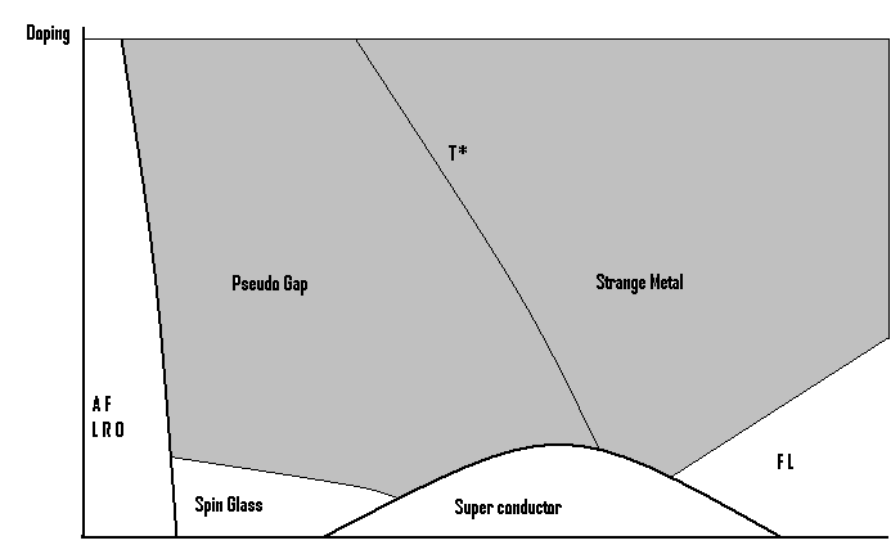

$\mathrm{T}$

FIG. 2: Qualitative phase diagram with in grey the "phases" considered in the paper

Dirac fermions with small Fermi surface $\left(\epsilon_{F} \sim t \delta\right)$ centered at the four nodes $\left( \pm \frac{\pi}{2}, \pm \frac{\pi}{2}\right)$, whereas in SM they exhibit a large Fermi surface $\left(\epsilon_{F} \sim t(1-\delta)\right)$, as expected from band structure calculations. A direct evidence of the small FS in PG might come from recent experiments on Shubnikov-de Haas oscillations [20].

Holons and spinons are gauge-invariantly coupled by a $U(1)$ slave-particle field, $A$, whose low energy effective action is obtained upon integration of the matter fields. As a consequence of the finite FS of holons the transverse gauge propagator exhibits a Reizer singularity [21] which dominates at large scales: for small $q, \omega, \omega /|\vec{q}|$

$$
\left\langle A_{\perp} A_{\perp}\right\rangle(\omega, \vec{q}) \sim\left(-\chi|\vec{q}|^{2}+i \kappa \omega /|\vec{q}|\right)^{-1},
$$

where $A_{\perp}$ is the transverse component of $A, \chi$ is the diamagnetic susceptibility and $\kappa$ the Landau damping. Both $\chi^{-1}, \kappa$ and the holon mass $m_{h}$ are $\sim \delta$ in PG and $\sim 1-\delta$ in SM. The scalar component $A_{0}$ has a low energy propagator given by

$$
\left\langle A_{0} A_{0}\right\rangle(\omega, q) \sim\left(\kappa\left(1+i \frac{\omega}{|\vec{q}|}\right) H(|\vec{q}|-|\omega|)+m_{0}^{2}\right)^{-1}
$$

where $m_{0}$ is a thermal mass generated by the spinons and $H$ the Heaviside step function. In view of the constant term in (2) the interaction mediated by $A_{0}$ is short ranged, hence subleading at large distance w.r.t. the interaction mediated by $A_{\perp}$. However, taking into account the renormalization of the transverse contribution discussed below, it gives the dominant contribution to $\gamma$ in PG for $T$ of the order of the holon Fermi temperature, which in this "phase" is rather small, of the order of a hundred $\mathrm{K}$ at low dopings. In the gauge correlator the momenta extend up to an UV cutoff $\Lambda \sim J$. 
As discussed in [4, 18, 22] at finite temperature $T$ the typical momentum scale of the transverse gauge fluctuations is given by the anomalous skin momentum $Q_{0}=(\kappa T / \chi)^{1 / 3}$. The interaction of holon and spinon modifies the gauge propagator, inducing a cutoff of the infrared momentum singularity, for $|q| \lesssim Q_{0}$, replacing $\kappa|\vec{q}|^{-1}$ by the sum of the conductivity of the spinongauge and holon-gauge subsystems, denoted by $\sigma_{s}$ and $\sigma_{h}$, respectively [23]. The splitting in high and low momenta contributions is explicitly realized in the calculations with a sharp cutoff at $|\vec{q}|=\zeta Q_{0}$, where $\zeta \simeq 0.4[25]$ and assuming a renormalization of $\kappa$ at high momenta accordingly.

To extract the entropy and the specific heat we start by computing the free energy $F$; then $S=-\frac{\partial F}{\partial T}$ and $\gamma=-\frac{\partial^{2} F}{\partial T^{2}}$, where we only differentiate w.r.t. the explicit dependence on $T$, thus ensuring $S(T=0)=0$. Within our approach $F$ is the sum of four terms: the contribution of free spinons, $F_{s}$, of free holons, $F_{h}$ and the fully renormalized contribution of transverse and scalar gauge fluctuations, $F_{\perp}, F_{0}$. Since the spinons are massive, the $T$-dependence of $F_{s}$ is negligible for $T$ lower than the spinon gap, which we estimate of the order of few hundreds K. For the holons we have the standard result (for each holon species)

$$
S_{h} \approx c m_{h} T
$$

where the phenomenological constant $c$ accounts also for the eccentricity of the FS due to neglected $t^{\prime}, t^{\prime \prime}$ terms. A comparison with [26] yields $c \approx 3$. We estimate $F_{\sharp} \sharp \sharp=\perp, 0$ following Ref. 27: if we denote by $D_{\sharp}$ the fully renormalized retarded Green function of the gauge field

$$
F_{\sharp} \sim \int d \omega \operatorname{coth}(\omega / 2 T) \int d^{2} q \arctan \left(\frac{\operatorname{Im} D_{\sharp}(\omega, \vec{q})}{\operatorname{Re} D_{\sharp}(\omega, \vec{q})}\right) .
$$

We remark that in the calculation of $F_{0}$ in [14] a sophisticated "ad hoc" regularization was needed because the euclidean scalar correlator vanishes in the limit of infinite frequency, thus making impossible a direct application of $\zeta$-function regularization, since both spinons and holons are gapless in the slave-boson approach. This problem does not arise here due to the constant term in the scalar correlator caused by gapful spinons. The key result of [14] that $F_{0}$ and $F_{\perp}$ have opposite sign is recovered here as a consequence of the opposite relative sign of the real and immaginary part of $D_{\sharp}$ for scalar and transverse components, as follows from eqs. (1) and (2).

The dominating contributions [28] to entropy of transverse gauge fluctuations turn out to be, up to logarithmic corrections,

$$
S_{\perp} \approx \begin{cases}Q_{0}^{2} \sim T^{2 / 3} m_{h}^{4 / 3} & \mathrm{PG} \\ Q_{0}^{2}, T \frac{\tilde{\sigma}}{\chi} H\left(Q_{0}^{2}-T \frac{\tilde{\sigma}}{\chi}\right) & \mathrm{SM}\end{cases}
$$

where $\tilde{\sigma}=\sigma_{h}+\sigma_{s} \sim \tau_{i m p}+\chi m_{s}^{2} T^{-1}[5]$. The $T^{2 / 3}$ behaviour is the standard one for $2 \mathrm{D}$ clean electrodynamics 27. The second contribution in SM comes from "small" momenta and is negligible in PG. The leading scalar contribution is given by

$$
S_{0} \approx-\frac{\Lambda}{v_{F}} T+\frac{1}{v_{F}^{2}} T^{2},
$$

where $v_{F}$ is the holon Fermi velocity. One can verify that increasing $T$ first the transverse then the scalar contribution dominates in PG, whereas in SM, in the temperature range considered the transverse contribution always dominates.

¿From equations (3), (5) and (6) one can easily derive the following consequences for the interpretation of experimental data within the spin-charge gauge approach:

1) The approximately linear behaviour of $S$ is basically due to the holons, although it is renormalized by gauge fluctuations. The increase of the slope at low $\delta$ and its saturation at higher $T$ or $\delta$ are due to the transition from $m_{h} \sim \delta$ characteristic of PG to $m_{h} \sim 1-\delta$ characteristic of SM.

2) The negative intercept of entropy is due to the scalar gauge contribution (6) in PG, negative in agreement with the general ideas discussed in the introductory section.

3) The upturn $\Delta \gamma^{L T}$ in $\gamma$ (and the analogous more evident in $S / T$ [2] ) is due to the contribution of transverse gauge fluctuations in PG. Their contribution in SM yields the decrease above $T_{\gamma}^{*}$, which we identify as the PG-SM crossover. These enhancement of entropy are due to the presence of the gapless transverse gauge mode.

4) The approximately linear increase of $\gamma$ in $\delta$ and in $T$, with almost $\delta$ independent slope, is due to the second term in (6) , presumably together with a pairing contribution (see below). This behavior replaces the linear slope due to the contribution of AF spin waves in the pristine material, now removed by the spinon gap.

More concretely a comparison between theory and experiments is summarized in figures 3 and 4, where in the inset the experimental data for selected dopings are plotted only for the region of parameters discussed above, where a comparison is meaningful. The theoretical curves have been obtained with the same value of the parameters used in [4, 5, 6] and by substituting in $\tilde{\sigma}$ the expression for the conductivities derived there. The value of $v_{F}$ in $\mathrm{PG}$ extracted from the slope of experimental $\gamma$ in the increasing range compared with the theoretical expression derived in from (6) turns out to be of the order of magnitude of the electron Fermi velocity found experimentally in ARPES [26] and of the holon Fermi velocity used in the calculation of transport properties in [4, 5], although 2-3 times smaller. Presumably this is due to a further contribution of pairing, as in the preformed pair (see e.g. [18, 29]) or fluctuating phase superconductor 30] approaches, which would yield a $T$ increasing density of states, not taken into account in the 

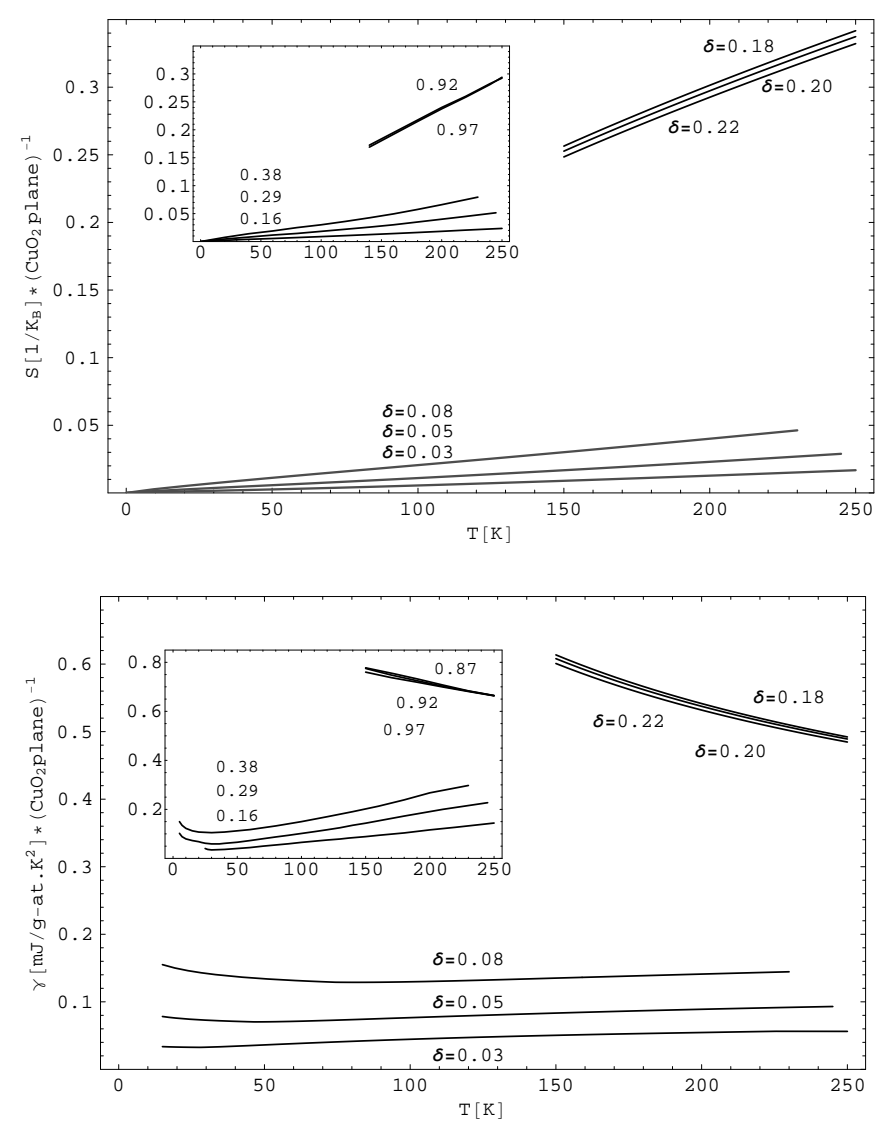

FIG. 3: The calculated temperature dependence for $S$ (above) and $\gamma$ (below) for selected dopings $\delta$ in both PG and SM "phases", in comparison with experimental data for analogous in-plane doping concentrations in $\mathrm{YBa}_{2} \mathrm{Cu}_{3} \mathrm{O}_{6+x}$ ), taken from [1] (inset; labels show $x$ ).

present simplified treatment. This increase should also account for the smooth transition from PG to SM discussed above in item 1). However a depletion mechanism of density of states (DOS), alone seems to be unable to reproduce the experimental behavior of $\gamma$, because lowering $T$ the curves at different dopings should converge near $T=0$ with increasing slope as $\delta$ increases (see e.g. [9], fig 12 ), whereas experimentally they are basically parallel at moderate temperatures with an upturn at low $T$ in nonsuperconducting samples. The presence of two distinct effects appears consistent also with recent experimental data on specific heat in $\mathrm{Bi}_{2} \mathrm{Sr}_{2-x} \mathrm{La}_{x} \mathrm{CuO}_{6}$ [31].

As one can see, even in our simplified treatment a qualitative agreement of the behavior both in $T$ and $\delta$ is found deeply in PG and SM in the region of validity discussed above, reproducing the features discussed in the introductory section. The derived behavior of the entropy in $\mathrm{SM}$ is also consistent with the numerical data extracted from the $t-J$ model with the Lanczos method 32] or high temperature expansion [14] in the overlapping range of temperature.

Summarizing, the reasonable agreement of doping-

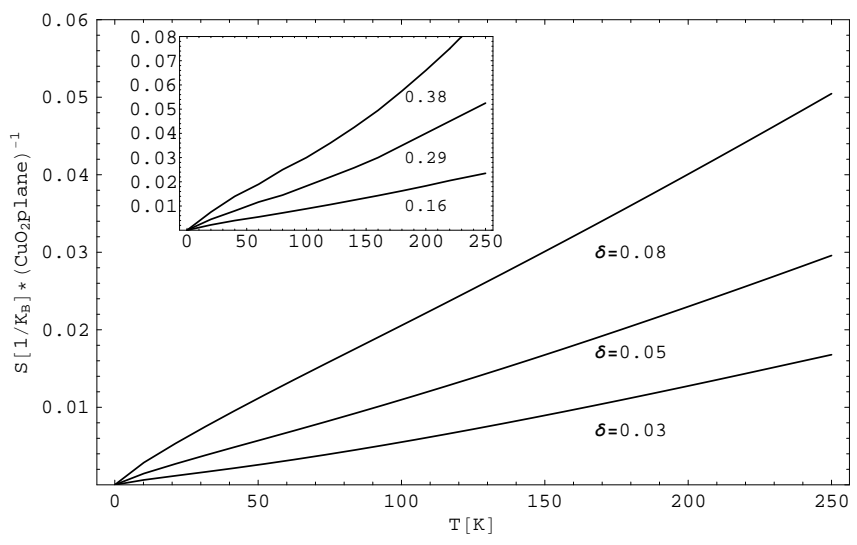

FIG. 4: Detail: Calculated $S$ in pseudogap "phase", in comparison with experimental data on YBCO (inset) taken from [1] with the same notations of Fig. 3

temperature dependence of experimental data and the theory discussed above suggests that one can interpret the low-temperature electronic specific heat in the normal state of hole-doped high Tc cuprates basically as due to fermionic holons, with small FS in the "pseudogap" and large FS in the "strange metal phase", but gauge fluctuations determine the variation in the $T$-dependence of the specific heat coefficient . Gauge fluctuations themselves reflect the changes in the spectrum of renormalized spin excitations and charge carriers.

In this approach the rather surprising negative intercept for the entropy is due to the negative contribution of scalar gauge fluctuations. Since the gauge field is just a consequence of the Gutzwiller projection, this interpretation in terms of a "constraint" gauge field suggests that this peculiar feature is rooted in the no-double occupancy condition, hence a basic characteristic of the low-energy description of doped Mott insulators. The phenomenon appears in PG due to the "effective" rather small Fermi temperature, a consequence of the $\pi$-flux, characteristic of a $2 \mathrm{D}$ system, suppressing the positive transverse contribution.

Furthermore, in this approach the gauge field is the "glue" binding spinon and holon into an electron resonance [5, 6]. The above interpretation then strengthens the idea of a composite nature of the charge carriers in the cuprates, an idea which is also strongly suggested by the metal-insulator crossover, as discussed in detail in [33].

Acknowledgments It is a pleasure for P.A.M. to thank Z.B. Su and L. Yu for the joy of a long collaboration and G. Orso for pointing out a mistake in an earlier version of the manuscript. Very useful discussions with N. Hussey, T.M. Rice, F. Toigo, T. Xiang and H.H Wen are also gratefully acknowledged. 
[1] J.W. Loram ,K.A. Mirza, J.R. Cooper, W.Y.Liang, Phys. Rev. lett. 711740 (1993)

[2] J. W. Loram et al. Physica C 235-240 134 (1994); J.W. Loram et al. J. Phys. Chem. Solids 5910 (1998)

[3] P.A. Marchetti, Z.B. Su, L. Yu, Phys. Rev. B 58, 5808 (1998).

[4] P.A. Marchetti, J.H. Dai, Z.B. Su and L.Yu, J. Phys. Condens. Matt. 12, L329 (2000)

[5] P.A.Marchetti, L. De Leo, G.Orso, Z.B. Su and L. Yu, Phys. Rev. B 69024527 (2004).

[6] P.A.Marchetti, G.Orso, Z.B. Su and L. Yu, Phys. Rev. B 71, 134510 (2005)

[7] N. Momono et al. Physica C 233395 (1994)

[8] Y. Ando, S. Komiya, K. Segawa, S. Ono, Y. Kurita, Phys. Rev. Lett.93 267001 (2004)

[9] J.W. Loram et al. J. Phys. Chem. Solids 6259 (2001)

[10] X.G. Wen and P.A. Lee, Phys. Rev. Lett. 76, 503 (1996); P.A. Lee, N. Nagaosa, T.K. Ng, X.G. Wen, Phys. Rev. B 57, 6003 (1998).

[11] D.H. Kim, P.A. Lee, and X.G. Wen, Phys. Rev. Lett. 79, 2109 (1997)

[12] P.Curty, H. Beck, Phys. Rev. Lett. 91257002 (2003)

[13] C.M. Varma, Phys. Rev. B 73, 155113 (2006)

[14] R. Hlubina, W. O. Putikka, T. M. Rice, and D. V. Khveshchenko Phys. Rev. B 46, 11224 (1992)

[15] J. Fröhlich and P.A. Marchetti, Phys. Rev. B 46, 6535 (1992).

[16] The coupling to the charge-gauge field induces a semionic Haldane statistics for holons allowing double occupation in $k$ space, therefore the density of states of the holon is twice the naive one; see P.A. Marchetti et al. in preparation and 33].
[17] R.J. Birgenau et al., Phys. Rev. B 38, 6614 (1988).

[18] P.A. Lee and N. Nagaosa, Phys. Rev. B 46 5621(1992).

[19] S. Chakravarty, R.B. Laughlin, D.K. Morr, C. Nayak, Phys. Rev. B 63, 094503 (2001).

[20] N. Doiron-Leyraud et al., Nature 447, 565 (2007); A. F. Bangura et al., Phys. Rev. Lett. 100, 047004 (2008); E. A. Yelland et al., Phys. Rev. Lett. 100, 047003 (2008).

[21] M.Y. Reizer, Phys. Rev. B 39, 1602 (1989); 40, 11571 (1989).

[22] L.B. Ioffe and P.B. Wiegmann, Phys. Rev. Lett. 65, 653 (1990).

[23] Notice that in view of the Ioffe-Larkin rule [24], holding in slave-particle gauge theories, the sum $\tilde{\sigma}=\sigma_{h}+\sigma_{s}$ is not equal to the physical conductivity, given by $\sigma=$ $\left(\sigma_{h}^{-1}+\sigma_{s}^{-1}\right)^{-1}$.

[24] L.B. Ioffe and A.I. Larkin, Phys. Rev. B 39, 8988 (1989).

$[25] \zeta$ is a constant $\mathrm{O}(1)$ fixed phenomenologically comparing with the experimental data

[26] T. Yoshida et al. Journal of Physics Condensed Matter 19, 125209 (2007)

[27] A.M. Tsvelik "Quantum Field Theory in Condensed Matter Physics" , Cambridge University Press 1995

[28] In the plot the full expression has been used.

[29] M. Randeria, Proceedings of the International School of Physics "Enrico Fermi" Varenna CXXXVI, ed G. Iadonisi et al. (Amsterdam, IOS Press, 1999); V. Emery and S. Kivelson, Nature 374, 434 (1995).

[30] M. Franz and Z. Tesanovic, Phys. Rev. Lett. 87, 257003 (2001).

[31] H.H. Wen, private communication.

[32] J. Jaklic and P. Prelovsek, Adv. Phys. 49, 1 (2000).

[33] P.A. Marchetti, Z.B. Su, L.Yu, J. Phys. Condens. Matter 19, 125209 (2007) 